VOL. 48 (1993) [141-149]

\title{
TWO GRAPH-COLOURING GAMES
}

\author{
Frank Harary and Zsolt Tuza
}

\begin{abstract}
We introduce two graph-colouring 2-person games, and analyse who has a winning strategy on some specific graphs such as the Petersen graph, and paths and cycles of given length.
\end{abstract}

\section{INTRODUCTION}

Recent research has attracted considerable interest in the study of the process of graph colouring. A typical example is 'on-line colouring' (see for example [2]). This concept can also be interpreted as a game during which Player A (the first player to move) presents the nodes of a graph $G$ one by one, with their adjacencies to the previously shown part of $G$, and Player $B$ colours the node just obtained. The colour of a node cannot be changed later. The aim of Player $B$ is to find a proper colouring with as few colours as possible. (A proper colouring of a graph $G$ is a colour assignment of the nodes in which no two adjacent nodes are monochromatic. We follow in general the notation and terminology of [3].)

The other main motivation for the present study is a recent work of Biro, Hujter, and the second author [1] in which the following problem is investigated:

Given an undirected graph $G=(V, E)$, a subset $V^{\prime} \subset V$ of nodes, a proper colouring $f^{\prime}$ on the subgraph induced by $V^{\prime}$, and an integer $k$, does there exist a proper colouring $f$ of the entire graph $G$ with at most $k$ colours, such that the restriction of $f$ to $V^{\prime}$ is $f^{\prime}$ ? As shown in [1], this question is closely related to many frequently-studied problems of graph theory, such as bipartite matching, perfect graphs, network flows, et cetera. (See also $[5,6]$.)

We now define two colouring games played on graphs by two players. In both of these games the input is a natural number $k$ and a (finite, undirected) graph $G=$ $(V, E)$ with node set $V$ and edge set $E$. Players $A$ and $B$ both know the whole input from the beginning of the game. They alternately assign colours from the set

\footnotetext{
Received 10 September 1992
}

Research of the first and second author was supported in part by grant N00014-90J1860 from the U.S. Office of Naval Research and by the 'OTKA' Research Fund of the Hungarian Academy of Sciences, respectively. We would also like to express our thanks to the organizers of the Julius Petersen Graph Theory Conference for local hospitality in Hindsgavl, Denmark, where part of this research was done in July 1990.

Copyright Clearance Centre, Inc. Serial-fee code: 0004-9729/93 \$A2.00+0.00. 
$\{1, \ldots, k\}$ to previously uncoloured nodes of $G$, so that the graph does not contain any monochromatic pair of adjacent nodes. The first move is made by Player $A$, and the game is over when no legal moves remain. The two games are

Achievement: The game is won by the player who can make the last move.

Avoidance: The game is lost by the player who makes the last move.

Since the input graph $G$ is finite, every game terminates after a finite number of steps, and therefore on each graph precisely one of Players $A$ and $B$ has a winning strategy. This fact leads to the following general question.

Problem 1: Characterise those graphs $G$ and integers $k$ for which

(a) Player A has a winning strategy in Achievement;

(b) Player A has a winning strategy in Avoidance.

We give a complete answer to Problem 1 in the cases when $G$ is a path or a cycle with $k=2$, or $G$ is the Petersen graph and $k=1,2,3$. Our results are summarised in Table 1. (The subscript $t$ always denotes the number of nodes in the path or cycle in question.) The small 'exceptional' cases are not indicated in the table, but they are easily seen: on $t \leqslant 2$ nodes every game terminates after precisely $t$ steps, and on the path $P_{3}$, Player A wins the Achievement game and loses the Avoidance game.

\begin{tabular}{|l|cc|}
\hline Graph and 'colour bound' $k$ & & Winner \\
& Achievement & Avoidance \\
\hline Petersen graph, $k=1$ & A & A \\
Petersen graph, $k=2$ & A & B \\
Petersen graph, $k=3$ & B & A \\
Path $P_{t}, t \geqslant 5$ odd & A & A \\
Path $P_{t}, t \geqslant 4$ even & B & B \\
Cycle $C_{t}, t \geqslant 3$ & B & A \\
\hline
\end{tabular}

Table 1. Winners on particular graphs; for paths and cycles, $k=2$.

\section{The Petersen graph}

The celebrated Petersen graph has been much studied. It seems appropriate to begin our study of these colouring games with this graph.

Theorem 1. When $G$ is the Petersen graph,

(1) for $k=1$, Player $A$ wins both Achievement and Avoidance; 
(2) for $k=2$, Player $A$ wins Achievement and Player $B$ wins Avoidance;

(3) for $k=3$, Player $B$ wins Achievement and Player $A$ wins Avoidance.

PROOF: $1^{\circ}$ It is well-known that $G$ is isomorphic to the complement of the line graph of the complete graph $K_{5}$ of order 5 . This fact is illustrated in Figure 1; a node labelled $i j(1 \leqslant i<j \leqslant 5)$ corresponds to edge $v_{i} v_{j}$ of $K_{5}$.

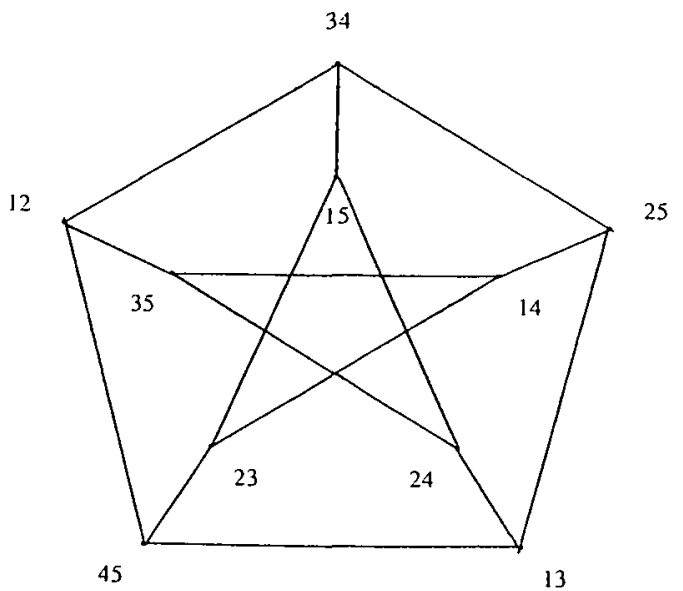

Figure 1. Representation of the Petersen graph as the complement of the line graph of $K_{5}$

Based on this isomorphism, there is a one-to-one correspondence between proper node colourings on subsets $V^{\prime} \subset V(G)$ and edge colourings on subsets $E^{\prime} \subset E\left(K_{5}\right)$ such that every monochromatic class $C$ of edges is intersecting, that is, $C$ is isomorphic to either the triangle $K_{3}$ or a star $S_{j}$ of $j$ edges $(1 \leqslant j \leqslant 4)$. An edge colouring of $K_{5}$ with this property will be called an intersecting colouring. We call an intersecting colouring an $i$-MIC (standing for Maximal Intersecting Colouring with $i$ colours) if it uses at most $i$ colours and cannot be extended to any other intersecting colouring of $K_{5}$ with at most $i$ colours.

Certainly, a colouring game terminates when a $k$-MIC has been constructed $(k=1,2,3)$.

$2^{\circ}$ For $k=1$, the first two steps are unique, as the two edges of colour 1 must intersect. If edges $v_{1} v_{2}$ and $v_{1} v_{3}$ have colour 1 , then Player A creates a triangle (and hence he wins Achievement) if he colours $v_{2} v_{3}$, and forces Player B to create an $S_{4}$ (so A wins Avoidance) if he colours $v_{1} v_{4}$.

$3^{\circ}$ Observe that a 2 -MIC induces $S_{3} \cup S_{4}$ or $K_{3} \cup S_{4}$ or $K_{3} \cup K_{3}$ (edge-disjoint 
union). Hence, in order to prove (2) it is sufficient to show that each player can force a 2-MIC in which at least one colour class is not a triangle. Certainly a player can do this if he can force a two-coloured triangle.

Say Player A assigns colour 1 to edge $v_{1} v_{2}$ in the first step. In Avoidance, Player $\mathrm{B}$ can create a two-coloured triangle if he assigns colour 2 to $v_{1} v_{3}$ in his first move. (Then, in his next turn, he can complete the colouring of the triangle on $\left\{v_{1}, v_{2}, v_{3}\right\}$, if Player A does not oblige.) It is also obvious that, in Achievement, Player A can create a two-coloured triangle in his second move if Player $\mathrm{B}$ colours an edge adjacent to $v_{1} v_{2}$ in his first move. Suppose that $v_{3} v_{4}$ is assigned colour 2 in the second step. Now Player A assigns colour 1 to $v_{2} v_{3}$, and then in his next move he can complete the colouring of the triangle on $\left\{v_{2}, v_{3}, v_{4}\right\}$ if Player B does not oblige. (If Player B assigns colour $i$ to some other edge in his second move, then colour $3-i$ is legal on $v_{2} v_{4}$.)

$4^{\circ}$ We prove that if a 3-MIC contains a monochromatic star $S_{4}$ then all the ten edges of $K_{5}$ are coloured.

Let $f$ be a 3 -MIC on $K_{5}$, with a monochromatic $S_{4}$. Then the restriction of $f$ to $K_{5}-E\left(S_{4}\right)$ is a 2 -MIC $f^{\prime}$ on $K_{4}$, say with node set $\left\{v_{i} \mid 1 \leqslant i \leqslant 4\right\}$. It suffices to show that each of the two colour classes of $f^{\prime}$ consists of three edges. (This will also imply that one of them is a triangle and the other is a star.)

Suppose first that $v_{1} v_{2}$ is the only edge of colour 1 in $f^{\prime}$. Then at most one of the edges $v_{1} v_{4}$ and $v_{2} v_{3}$ can have colour 2 , so the other can be assigned colour 1 . Thus, $f^{\prime}$ is not maximal, a contradiction. Next, suppose that the first colour class consists of just two edges, say $v_{1} v_{2}$ and $v_{1} v_{3}$. Then, again, at most one of the edges $v_{1} v_{4}$ and $v_{2} v_{3}$ can have colour 2 , so that the other can be assigned to colour 1 . Thus, $f^{\prime}$ is not maximal.

$5^{\circ}$ We prove that if a $3-\mathrm{MIC}$ contains a monochromatic triangle then precisely eight or ten edges of $K_{5}$ are coloured.

Let $f$ be a $3-\mathrm{MIC}$ on $K_{5}$ and suppose that colour 3 induces a triangle $\left\{v_{3}, v_{4}, v_{5}\right\}$. Then the restriction of $f$ to $K_{5}-E\left(K_{3}\right)$ is a 2-MIC. If colour 1 is a triangle then colour 2 induces a maximal star in a cycle of length four, implying that precisely two edges of $K_{5}$ remain uncoloured in $f$. If each of colours 1 and 2 induces a (non-extendable) star, then one of those stars has $v_{1}$ (or $v_{2}$ ) as its centre, for otherwise the edge $v_{1} v_{2}$ could be assigned to colour 1 or 2 , contradicting the maximality of $f$. Hence, the number of uncoloured edges is equal to zero or two, according to whether the other centre is or is not identical to $v_{2}$, respectively.

$6^{\circ}$ Next, we show that either player can force a monochromatic $K_{3}$ or $S_{4}$. In the proof below we use the following shorthand: $f(a, b)=c$ means that the next player assigns colour $c$ to the edge $v_{a} v_{b} ;(K i)$ and $(S i)$ abbreviate that a $K_{3}$ or $S_{4}$ of colour $i$ is constructed (or can be forced in the next step where $\Rightarrow$ is written). 
The strategy of Player A is

$$
\begin{array}{llll}
f(1,2)=1-f(1,3)=1 ; & f(2,3)=1 \quad(K 1) & \\
f(1,2)=1-f(1,3)=2 ; & f(2,3)=3-f(1,4)=1 ; & f(2,4)=1 \quad(K 1) \\
f(1,2)=1-f(3,4)=2 ; & f(2,3)=3-f(1,3)=1 ; & f(1,4)=1 \Rightarrow \quad(S \\
f(1,2)=1-f(3,4)=2 ; & f(2,3)=3-f(1,3)=2 ; & f(1,4)=2 \quad(K 2) \\
f(1,2)=1-f(3,4)=2 ; & f(2,3)=3-f(1,3)=3 ; & f(1,5)=1 \Rightarrow \quad(K 1) \\
f(1,2)=1-f(3,4)=2 ; & f(2,3)=3-f(1,4)=1 ; & f(1,3)=1 \Rightarrow \quad(S 1) \\
f(1,2)=1-f(3,4)=2 ; & f(2,3)=3-f(1,5)=1 ; & f(2,5)=1 \quad(K 1) \\
f(1,2)=1-f(3,4)=2 ; & f(2,3)=3-f(2,5)=1 ; & f(1,5)=1 \quad(K 1) \\
f(1,2)=1-f(3,4)=2 ; & f(2,3)=3-f(2,5)=3 ; & f(3,5)=3 \quad(K 3)
\end{array}
$$

Apart from permutations of colours and nodes, these sequences cover all possibilities, hence Player A can always force a monochromatic star or triangle.

The strategy of Player B is even simpler. After the two moves

$$
f(1,2)=1-f(1,3)=1
$$

Player A has six possible moves $(f(2,3)=1, f(1,4)=1, f(2,3)=2, f(1,4)=2$, $f(2,4)=2, f(4,5)=2)$. In the latter three, however, Player B immediately creates a monochromatic triangle, assigning $f(2,3)=1$, and in the first one the colour-1 edges themselves form a triangle. In the second and third cases an efficient strategy is

$$
\begin{array}{ll}
f(1,4)=1-f(1,5) \quad(S 1) & \\
f(2,3)=2-f(4,5)=3 ; & f(1,4)=1-f(1,5)=1 \\
f(2,3)=2-f(4,5)=3 ; & f(1,4)=3-f(1,5)=3 \\
f(2,3)=2-f(4,5)=3 ; & f(2,4)=2-f(3,4)=2 \\
f(2,3)=2-f(4,5)=3 ; & f(2,4)=3-f(2,5)=3
\end{array}
$$

Hence, Player B can also force a monochromatic star or triangle.

$7^{\circ}$ To complete the proof of the theorem, in Avoidance Player A (in Achievement Player $\mathrm{B}$, respectively) chooses the strategy described in $6^{\circ}$ to force a monochromatic $K_{3}$ or $S_{4}$. Then, by $4^{\circ}$ and $5^{\circ}$, each game terminates after an even number of moves, that is, the last move is done by Player B. Hence Player A wins Avoidance and loses Achievement. 


\section{Paths}

The aim of this section is to solve Problem 1 for paths, when two colours can be used. Some of the observations will be applied in the next section as well, where the corresponding question will be settled for cycles. Here we prove

Theorem 2. Let $G$ be the path with $t$ nodes, and let $k=2$. Then for $t \geqslant 5$ odd, Player $A$ - and, for $t \geqslant 4$ even, Player $B$ - has a winning strategy in both Achievement and Avoidance.

Proof: $1^{\circ}$ Let $G$ have nodes $v_{1}, v_{2}, \ldots, v_{t}$ in this order. First we describe two simple winning strategies in Achievement, according to the parity of $t$.

Assume first that $t$ is even. If Player $A$ assigns colour $i \in\{1,2\}$ to node $v_{j}$ in some move, then the answer of Player B is to assign colour $3-i$ to node $v_{t+1-j}$. Since $v_{(t / 2)+1}$ and $v_{t / 2}$ will be asigned opposite colours, every such move of Player B is legal. Thus, the game terminates after an even number of steps, implying that Player $B$ can win Achievement for every even $t \geqslant 2$.

If $t$ is odd, then the strategy of Player $A$ is to maintain a 'symmetric' colouring in every step. For this purpose, he assigns colour 1 to the middle node $v_{(t+1) / 2}$ in the first step. Then, if Player B has assigned colour $i \in\{1,2\}$ to node $v_{j}$ in some move, the answer for Player $\mathrm{A}$ is to assign colour $i$ to node $v_{t+1-j}$. Obviously, this game terminates after an odd number of steps, implying that Player A can win Achievement for every odd $t \geqslant 3$.

$2^{\circ}$ Now we consider the Avoidance game. The winning strategy requires some preliminary observations. A leaf of a tree is an endnode. We prove the following statement.

Lemma 1. Let $T$ be a path with $m \geqslant 0$ internal nodes, and with only both leaves coloured. If the leaves have distinct colours (the same colour, respectively), then each player can force the number of uncoloured nodes in $T$ at the end of the game to have the same parity (opposite parity, respectively) as $m$.

Proof of LEMMA 1: The proof goes by induction on $m$. For $m \leqslant 3$, the validity of the claim is obvious. Note further that on colouring an internal node next to an endnode of $T$ in the first step, the parity of the length of the uncoloured path-as well as the colour assigned to one of its ends- changes. Hence, the requirement on the parity of uncoloured nodes at the end of the game remains the same. Thus Player A has a strategy on $m$ nodes satisfying the requirements of the lemma if Player $B$ has one on $m-1$ nodes.

Now we describe a strategy for Player $B$, with the assumption that the game is played on $T$ only. Consider $m \geqslant 4$. Then every internal node $x$ has a neighbour $y$ whose other neighbour $z$ is not a leaf of $T$. If Player A assigns colour $i$ to node $x$ then 
Player B assigns colour $3-i$ to $y$. This can be done since $z$ is uncoloured.

To show that this is an efficient strategy for Player $B$, observe that after such a pair of moves, the $m-2$ uncoloured nodes form two paths with, say, $m^{\prime}$ and $m^{\prime \prime}$ internal nodes, $m^{\prime}+m^{\prime \prime}=m-2$ (where $m^{\prime \prime}=0$ is possible). Since $x$ and $y$ are assigned distinct colours, precisely one of these two paths has identical colours on its two leaves if and only if the leaves of $T$ have the same colour. Note further that precisely one of $m^{\prime}$ and $m^{\prime \prime}$ is odd if and only if $m$ is odd.

If the leaves of $T$ have distinct colours then by the induction hypothesis Player $B$ can force the numbers of uncoloured nodes on the two paths to be congruent either to $m^{\prime}$ and $m^{\prime \prime}(\bmod 2)$ or to $m^{\prime}-1$ and $m^{\prime \prime}-1(\bmod 2)$. In either case, their total number has the same parity as $m$. On the other hand, if the leaves of $T$ have the same colour, then the numbers of uncoloured nodes can be forced to be congruent either to $m^{\prime}$ and $m^{\prime \prime}-1(\bmod 2)$ or to $m^{\prime}-1$ and $m^{\prime \prime}(\bmod 2)$. Hence, the parity of their total number is equal to that of $m-1$, and the validity of Lemma 1 follows.

$3^{\circ}$ Continuing with the proof of the theorem, we now consider the case when $t$ is odd. To win A voidance, Player A first assigns colour 1 to $v_{(t+1) / 2}$. Set $L=\left\{v_{1}, v_{2}\right\}$ and $R=\left\{v_{t-1}, v_{t}\right\}$. Then Player A follows the 'symmetric' strategy described in $1^{\circ}$ until Player B colours some node of $L \cup R$. When Player B visits a node of $L \cup R$ for the first time during the game, say he assigns colour $i$ to node $v_{j}$, then Player A assigns colour $3-i$ to node $v_{t+1-j}$ if possible.

If this is a legal move then from now on Player A applies the strategy described in the proof of Lemma 1 on each uncoloured subpath between $v_{j}$ and $v_{t+1-j}$. The lengths of these subpaths kelow and above $v_{(t+1) / 2}$ are in one-to-one correspondence, by the symmetric arrangement of coloured nodes. The only difference on the two sides is that precisely one of the two uncoloured subpaths starting at $v_{j}$ and $v_{t+1-j}$ inside the 'interval' $\left[v_{j}, v_{t+1-j}\right]$ has identical colours on its two leaves. Thus, by Lemma 1 , Player A can force an odd number of uncoloured nodes in $\left[v_{j}, v_{t+1-j}\right]$. It is trivial that $(L \cup R)-\left[v_{j}, v_{t+1-j}\right]$ - which is either $\emptyset$ or $\left\{v_{1}, v_{t}\right\}$ - will be coloured completely at the end, that is, the game terminates after an even number of steps. Hence, Player A wins Avoidance for $t$ odd whenever colour $3-i$ can be assigned to $v_{t+1-j}$.

In the case when colour $3-i$ cannot be assigned to $v_{t+1-j}$, we must have $j=2$ or $j=t-1$ (and $v_{3}$ as well as $v_{t-2}$ has colour $3-i$ ); say, $j=2$. Then Player A assigns colour $i$ to $v_{t}$ and applies the strategy described above on the interval $\left[v_{2}, v_{t}\right]$. Hence, at the end of the game an odd number of nodes will be uncoloured in $\left[v_{2}, v_{t}\right]$. Clearly, $v_{1}$ will be coloured, so Player A wins Avoidance in this case as well.

$4^{\circ}$ To win Avoidance for $t$ even, Player B applies the 'antisymmetric' strategy given at the beginning of the proof, until Player A visits $L \cup R$. Then, if Player $A$ assigns colour $i$ to some $v_{j} \in L \cup R$, the answer for Player B is to assign colour $i$ to 
$v_{t+1-j}$ if possible. Otherwise, if say $v_{2}$ has colour $i$, then assign $v_{t}$ to colour $3-i$. Again, it follows from Lemma 1 that Player $B$ can force an odd number of uncoloured nodes in $\left[v_{2}, v_{t}\right]$ and in $G$, too, that is, he wins Avoidance for $t$ even.

REMARK. Note that great similarity between the strategy of $1^{\circ}$ and that for the Latin Square Achievement Game by Leary and one of us, [4].

\section{CyCles}

We now handle these games for the family of cycles.

Theorem 3 . Let $G$ be a cycle with $t \geqslant 3$ nodes, and let $k=2$. Then Player $A$ has a winning strategy in Avoidance and Player $B$ has a winning strategy in Achievement.

Proof: $1^{\circ}$ Let $G$ have nodes $v_{1}, v_{2}, \ldots, v_{t}, v_{1}$ in this cyclic order, and assume that Player A assigns colour 1 to node $v_{1}$ in the first step. Then Player B wins Achievement if he assigns colour 2 either to $v_{2}$ (if $t$ is even) or to $v_{3}$ (if $t$ is odd), and applies his 'antisymmetric' strategy described in part $1^{\circ}$ of the proof of Theorem 2 , on the $t-2$ or $t-3$ uncoloured nodes viewed as a path of even order.

$2^{\circ}$ For $t=3,4$, it is easy to see that every game terminates after an even number of steps, that is, Player A wins Avoidance in these cases. To win for $t \geqslant 5$, in his second move Player $A$ assigns a colour to some node in such a way that he creates two nodes $x, y$ of distinct colours, with the following property: If $t$ is even then $x$ and $y$ are adjacent, and if $t$ is odd then $x$ and $y$ have an uncoloured neighbour in common. This situation can always be ensured (for $t \geqslant 5$ ), independently of the first move of Player B.

Denote by $z$ the third node (distinct from $x$ and $y$ ) coloured so far. Its colour is identical to the one of $x$ or $y$, but not both. Moreover, the parities of the $x-z$ and $y-z$ paths are distinct, regardless the value of $t$. Thus, by Lemma 1, Player $A$ can force for these two paths together an even number of uncoloured nodes at the end of the game. There is one further uncoloured node - the common neighbour of $\boldsymbol{x}$ and $y$-when $t$ is odd. Consequently, Player A can always ensure an even number of steps, and hence he wins Avoidance.

\section{CONCLUDING REMARKS}

It is immediate from the definitions that if the number $k$ of colours is greater than the maximum degree of the input graph $G=(V, E)$, then Achievement-as well as Avoidance-terminates when all nodes are coloured. Hence, the winner is determined by the parity of $n=|V|$, independently of the actual moves during the game. For this reason, the most interesting particular cases of Problem 1 seem to be

(1) $k=$ the maximum degree of $G$, 
(2) $k=$ the chromatic number of $G$,

(3) $k=1$.

In (3), a game terminates when a maximal independent set is found. It remains an open problem to analyse the Achievement and Avoidance games on paths and cycles of $n$ nodes when $k=1$.

\section{REFERENCES}

[1] M. Biró, M. Hujter and Zs. Tuza, 'Precoloring extension I. Interval graphs', Discrete Math. 100 (1992), 267-279.

[2] A. Gyárfás and J. Lehel, 'On-line and first-fit colourings', J. Graph Theory 12 (1988), 217-227.

[3] F. Harary, Graph theory (Addison-Wesley, Reading, 1969).

[4] F. Harary and C. Leary, 'Latin square achievement games', J. Recreational Math. 16 (1983-84), 241-246.

[5] M. Hujter and Zs. Tuza, 'Precoloring extension II. Graph classes related to bipartite graphs', Acta Math. Univ. Comenian. (1993) (to appear).

[6] M. Hujter and Zs. Tuza, 'Precoloring extension III. Classes of perfect graphs', (submitted).

Department of Computer Science

New Mexico State University

Las Cruces NM 88003

United States of America
Computer and Automation Institute

Hungarian Academy of Sciences

H-1111 Budapest

Kende u. 13-17

Hungary 